\title{
Comparação de Duas Diferentes Intensidades de Alongamento na Amplitude de Movimento
}

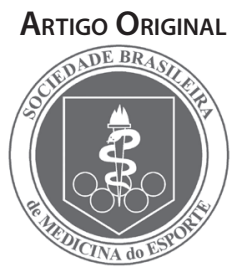

\section{Comparison of Two Different Stretching Intensities in the Range of Motion}

\author{
Mauro Heleno Chagas' \\ Elder Lopes Bhering \\ Juliana Castro Bergamini' \\ Hans-Joachim Menzel \\ 1. Escola de Educação Física, \\ Fisioterapia e Terapia Ocupacional \\ da Universidade Federal de Minas \\ Gerais (EEFFTO/ UFMG)
}

\section{Endereço para correspondência:} Prof. Dr. Mauro Heleno Chagas Escola de Educação Física,

Fisioterapia e Terapia Ocupacional - EEFFTO/UFMG

Av. Presidente Carlos Luz 4664, Pampulha - CEP: 31310-250

Belo Horizonte - Minas Gerais

Email: mauro@eef.ufmg.br

Submetido em 13/02/2007

Versão final recebida em 14/04/2007

Aceito em 13/06/2007

\begin{abstract}
RESUMO
O alongamento é uma prática comum no treinamento da flexibilidade, porém existe uma deficiência de informações específicas sobre os componentes da carga utilizados, em especial, sobre a intensidade do alongamento. O presente estudo teve como objetivo investigar o efeito agudo de duas diferentes intensidades de alongamento na amplitude de movimento (ADM) de extensão do joelho. Participaram deste estudo 14 voluntários do sexo masculino. Os dois membros inferiores de cada participante foram avaliados, sendo que um membro executou o treinamento na intensidade submáxima de alongamento (TS, N=14) e o outro na intensidade máxima de alongamento ( $\mathrm{TM}, \mathrm{N}=14)$. $\mathrm{O}$ treinamento utilizou a técnica passiva-estática executando quatro repetições com duração de 15 segundos. Foi encontrada diferença estatisticamente significativa para os valores de ADM entre o pré-teste $(68,0 \pm 10,5)$ e o pós-teste $(74,0 \pm 10,0)$ no TM. Os valores de ADM para o pré-teste $(68,9 \pm 14,0)$ e o pós-teste $(71,0 \pm 14,4)$ no TS não apresentaram diferença significativa. Os resultados analisados através da ANOVA two-way apresentaram interação significativa entre o grupo e o teste $(F=1.26$; $d f=8,62 ; p=0,007)$. $O$ estudo demonstrou que o treinamento na intensidade máxima de alongamento promove aumento agudo da ADM. Dessa forma, os estudos que avaliam os componentes ideais da carga de treinamento na melhora da flexibilidade não devem negligenciar o controle da intensidade do estímulo de alongamento.
\end{abstract}

Palavras-chave: flexibilidade, treinamento, biomecânica.

\begin{abstract}
Stretching is a usual practice in flexibility training; however, specific information on load is still scarce, mainly about what concerns the stretching intensity. The aim of the present study was to investigate the acute effect of two stretching intensities in the range of motion (ROM) of knee extension, after a single training session. Fourteen males participated in this study. Both lower limbs of each volunteer were assessed, being one leg submitted to a submaximal stretching program (SSP) and the other to a maximal stretching program (MSP). The training session consisted of four static-passive hamstring stretching repetition of fifteen seconds each. MSP exhibited a significant difference between pre-test (68.0 \pm 10.5$)$ and post-test $(74.0 \pm 10.0)$ to the ROM figures. However, significant difference was not found for the pre-test (68.8 \pm 14.0$)$ and post-test (70.9 \pm 14.4$)$ for the SSP. The results analyzed through two-way ANOVA method showed significant interaction between the group and the test $(F=1.26 ; \mathrm{df}=8.62 ; \mathrm{p}=0.007)$. These findings demonstrated that the maximal stretching program causes an acute increase at ROM. Thus, the studies to assess the ideal training load components in order to improve flexibility should not take the stimulus intensity control for granted.
\end{abstract}

Keywords: flexibility, training, biomechanics.

\section{INTRODUÇÃO}

A prática de exercícios visando a melhora da flexibilidade apresenta seus benefícios descritos na literatura ${ }^{(1,2)}$. As técnicas de alongamento mais utilizadas no treinamento da flexibilidade são: Balística ou Dinâmica, Estática e Facilitação Neuromuscular Proprioceptiva $(\text { FNP) })^{(3,4)}$

A eficiência dessas técnicas é freqüentemente investigada utilizando-se, como parâmetro, alterações na amplitude de movimento $(A D M)^{(5)}$. Apesar da técnica Estática ser largamente empregada nos programas de treinamento da flexibilidade devido a sua simplicidade de execução ${ }^{(6,7)}$, alguns estudos demonstraram a superioridade de técnicas de FNP em relação às técnicas passivas e dinâmicas no aumento da $\mathrm{ADM}^{(7-12)}$. Outros estudos, no entanto não confirmaram tal diferença $a^{(2,13-18)}$. A utilização de diferentes procedimentos na mensuração da ADM e nas configurações da carga de treinamento pode explicar a variabilidade dos resultados ${ }^{(4,5,19)}$. Não há consenso na literatura sobre a configuração dos componentes da carga de treinamento da flexibilidade que possibilite maiores ganhos de $\mathrm{ADM}^{(1,20)}$. Isso é demonstrado no que diz respeito ao componente duração(20-22) e à relação número de repetições - duração ${ }^{(4,20,23)}$. Além disso, estudos sobre a relevância da intensidade do estímulo de alongamento para o desenvolvimento da flexibilidade ainda são escassos ${ }^{(24)}$. 
A intensidade do estímulo é considerada um componente da carga de treinamento essencial para a determinação das adaptações desejadas no treinamento da força muscular(25-27), mas ainda não está claro se esse componente é igualmente importante para a flexibilidade. Marschall(24) comparou o efeito agudo de 15 repetições passivas-dinâmicas realizadas em duas diferentes intensidades de alongamento (máxima e submáxima) sobre o aumento da ADM. Nesse estudo foi observado um aumento significativo da ADM para as duas intensidades de alongamento comparando-se o pré e pós-treinamento, porém o aumento da ADM foi significativamente maior no pós-treinamento para a intensidade máxima de alongamento. Uma evidência indireta de que a intensidade de estímulo de alongamento pode ser fundamental para a alteração da ADM foi apresentada por Sullivan et al. ${ }^{(16)}$. Esses autores investigaram o efeito de duas diferentes posições do quadril na execução do alongamento dos posteriores da coxa no aumento da ADM. Os resultados mostraram que o exercício que proporcionava uma maior tensão de alongamento na musculatura (maior intensidade de alongamento) foi significativamente mais efetivo para aumentar a ADM do que o exercício que estimulava a musculatura com menor tensão de alongamento independentemente da técnica realizada para o treinamento da flexibilidade. Isso demonstra que a intensidade do estímulo de alongamento pode influenciar as adaptações relacionadas a ADM. Desconsiderar o controle desta variável pode conduzir a interpretações equivocadas sobre o efeito da carga de treinamento da flexibilidade.

Outro aspecto importante é a relação entre a intensidade do alongamento e a técnica utilizada. É necessário verificar se os resultados encontrados por Marschall(24), utilizando a técnica passiva-dinâmica, ocorrem também quando outras técnicas, como a passiva-estática, são realizadas.

Sendo assim, o presente estudo teve como objetivo comparar os efeitos do alongamento passivo-estático realizado em duas intensidades de alongamento, máxima e submáxima, no aumento da ADM.

\section{MATERIAS E MÉTODOS}

\section{Amostra}

Participaram nesta pesquisa 14 estudantes de Educação Física do sexo masculino, com idade média de 23,1 anos $( \pm 2,3)$, massa corporal média de $74,7 \mathrm{~kg}( \pm 12,0)$ e estatura média de $175,9 \mathrm{~cm}( \pm 6,6)$. Foram incluídos os indivíduos que não apresentavam doenças em membros inferiores ou coluna lombar e que demonstraram encurtamento dos músculos posteriores da coxa. A definição funcional de encurtamento no presente estudo foi o ângulo de extensão de joelho menor ou igual a $85^{\circ}$, para o teste de extensão de joelho modificado realizado no estudo, partindo da posição inicial pré-estabelecida (quadril e joelho flexionados a $90^{\circ}$ ). Nenhum exercício de alongamento foi permitido antes da coleta dos dados.

O estudo e seu procedimento foram aprovados pelo Comitê de Ética em Pesquisa da UFMG (ETIC 318/06). Todos os participantes foram previamente esclarecidos sobre os objetivos e procedimentos referentes à pesquisa e assinaram um termo de consentimento livre e esclarecido, concordando em participar da pesquisa.

\section{Procedimento}

O estudo foi realizado em três etapas: avaliação da ADM submáxima e máxima, sessão de treinamento e reavaliação da ADM máxima (FIG.1).

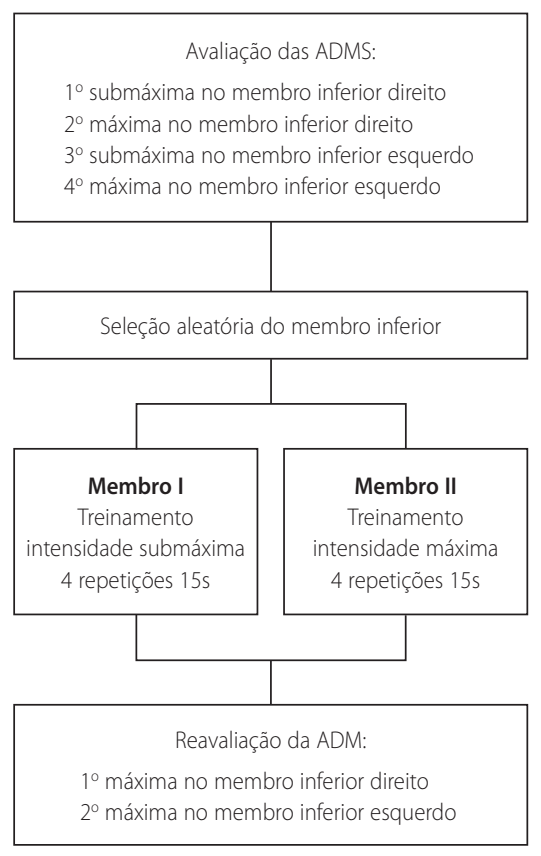

Figura 1. Apresentação esquemática de procedimento de coleta

A ADM submáxima foi avaliada no pré-teste com o objetivo de estabelecer a intensidade de alongamento submáxima no treinamento. A ADM submáxima foi mensurada antes da ADM máxima na tentativa de criar um padrão para a avaliação e diminuir possíveis interferências na sua identificação. É esperado que o estímulo maior de alongamento da ADM máxima possa gerar alguma adaptação e assim interferir na percepção subjetiva conseqüente da ADM submáxima.

Apenas a ADM máxima foi utilizada na avaliação dos diferentes treinamentos, pois a mesma apresenta dados mais consistentes de confiabilidade, para o instrumento utilizado, na avaliação do desempenho de flexibilidade. Um estudo prévio investigou a confiabilidade da mensuração da ADM máxima utilizando o mesmo procedimento da presente pesquisa e foram registradas correlações altas e significativas tanto para a forma passiva $(r=0,93 ; n=60)$ como para ativa $(r=0,94$; $n=60$ ) de mensuração da ADM máxima para um intervalo entre as medidas de 48 horas.

\section{Avaliação das ADMs submáxima e máxima}

Para a realização do teste de extensão de joelho modificado (TEJ-m) ${ }^{(28)}$ foi utilizada uma maca adaptada contendo um rolo central, quatro fixadores do tronco e uma cinta com velcro. O rolo central garante a manutenção do quadril na posição de $90^{\circ}$ de flexão através do contato com a coxa, sendo sustentado por duas estruturas verticais ajustáveis a diferentes comprimentos do membro. O rolo ainda apresenta, na sua parte superior, uma escala métrica graduada em centímetros para verificar e controlar o movimento de adução ou abdução do quadril.

Os quatro fixadores de tronco, reguláveis horizontalmente, ajustamse às pessoas de diferentes estaturas. Dois dos fixadores encontram-se na altura dos ombros (um de cada lado) e dois na altura dos quadris (um de cada lado). Para garantir que a fixação do tronco fosse repetida com exatidão durante o procedimento de coleta de dados uma escala métrica graduada em centímetros foi posicionada paralela ao eixo de deslocamento desses fixadores. 
O voluntário foi posicionado em decúbito dorsal sobre a maca e o membro inferior a ser testado fixado a $90^{\circ}$ de flexão do quadril. Essa angulação foi padronizada através de um esquadro, alinhando verticalmente o epicôndilo lateral e o trocânter maior do fêmur. O rolo central foi regulado de forma que o contato com o membro avaliado sempre ocorresse na parte distal anterior da coxa, acima da patela. Em seguida os fixadores do ombro e do quadril foram ajustados de modo a restringir o deslocamento horizontal do sujeito na maca. Uma cinta de velcro foi fixada na parte distal da coxa do membro inferior não testado, a aproximadamente cinco centímetros acima da borda superior da patela, mantendo o membro inferior estendido e assim evitando uma possível compensação que favorecesse o movimento de flexão do joelho e retroversão da pelve.

Para estabelecer a angulação de $90^{\circ}$ de flexão da articulação do joelho, posição na qual o flexômetro estaria marcando zero graus, utilizou-se um goniômetro universal. Esse instrumento foi posicionado de forma que o braço fixo seguia o alinhamento do trocânter maior do fêmur ao epicôndilo lateral do fêmur e o braço móvel seguia o alinhamento do maléolo lateral ao epicôndilo lateral do fêmur.

A ADM submáxima foi definida como o momento em que o voluntário percebesse o início do alongamento dos músculos posteriores da coxa, ou seja, a primeira sensação que a musculatura posterior da coxa estivesse sendo alongada ${ }^{(24)}$. A ADM máxima foi considerada como a máxima tolerância ao exercício de alongamento, ou seja, a máxima amplitude de movimento que o avaliado pudesse supor$\operatorname{tar}^{(24)}$. As informações foram transmitidas ao voluntário através da leitura padronizada das definições de cada ADM e realizada pelo avaliador A, antes da coleta de dados. Sendo assim a execução do teste para as ADMs foi finalizada pelo próprio indivíduo pronunciando-se verbalmente.

Cada membro inferior foi avaliado separadamente, sendo executadas três medidas. A primeira adotada como familiarização e a média das outras duas foi utilizada para o desempenho no teste. O tempo entre uma medida e outra era aquele necessário para retornar à posição inicial e recomeçar a próxima medida. As mensurações iniciavam-se sempre pelo membro inferior direito e pela ADM submáxima.

Após o posicionamento do voluntário, um flexômetro (Leighton; modelo 01146) foi fixado cinco centímetros acima do maléolo na região do tornozelo para a mensuração das ADMs. Partindo da posição inicial, uma extensão passiva do joelho foi realizada pelo avaliador A, lentamente (FIG. 2). Ao atingir a posição final para cada ADM foi realizada a leitura da mensuração do grau de extensão.

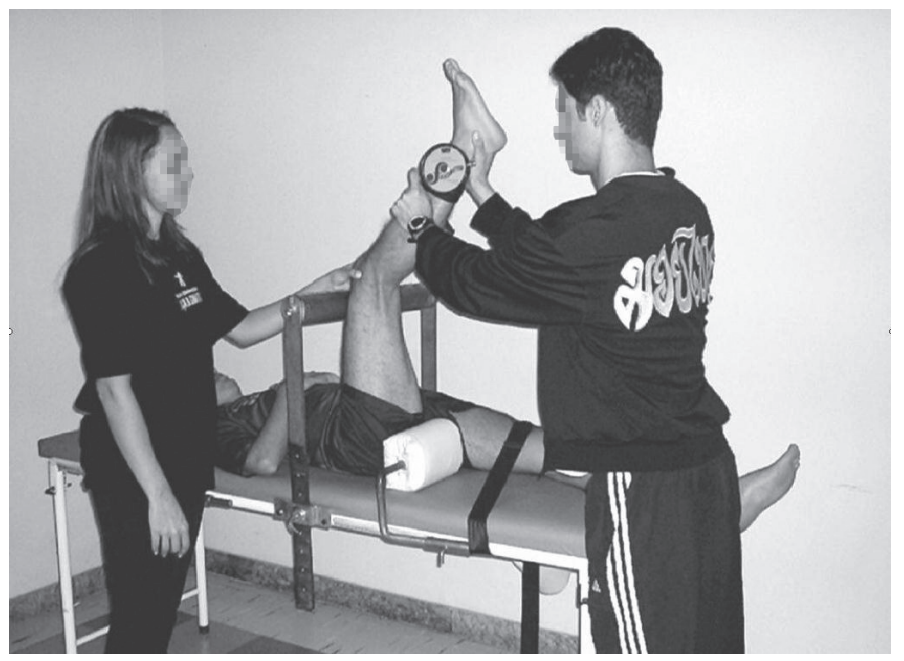

Figura 2. Execução do TEJ-modificado
O grau de confiabilidade das medidas para as ADMs foi determinado por um estudo prévio utilizando dois procedimentos. Um deles foi o coeficiente de correlação de Pearson, sendo ele de 0,94 para a medida de ADM máxima e de 0,97 para a ADM submáxima em um mesmo dia ${ }^{(29)}$. Outro procedimento foi à comparação das médias utilizando o teste-t de Student para amostras pareadas num intervalo de 48 horas. Não foi observada diferença significativa entre os valores médios de ADM articular para a intensidade submáxima $(p=0,70)$ e máxima $(p=0,28)^{(29)}$, indicando a baixa variabilidade das medidas. Imediatamente após os procedimentos de avaliação das ADMs foi iniciada a sessão de treinamento.

\section{Sessão de Treinamento}

Utilizando a técnica de alongamento passiva-estática foi realizada uma única sessão de treinamento, na qual os indivíduos executaram quatro repetições com duração de 15 segundos. Essa configuração da carga baseou-se em pesquisas que demonstraram um aumento da ADM nestas condições ${ }^{(30-33)}$. O treinamento foi realizado em ambos os membros inferiores sem intervalo entre as repetições e executado na própria maca utilizada para o TEJ-m. O avaliador A executava, a partir da posição inicial do TEJ-m, a extensão do joelho apoiando uma mão no calcâneo, permitindo que a articulação do tornozelo estivesse relaxada, e a outra mão na perna em sua região lateral acima do flexômetro.

A ADM executada durante o treinamento submáximo permaneceu constante em todas as quatro repetições, sendo padronizada pela média dos valores do pré-teste da ADM submáxima. Já, no grupo de treinamento máximo, a ADM permaneceu livre nas quatro repetições, pelo fato que o avaliado poderia sempre atingir a máxima amplitude de movimento por tentativa. Com um membro foi executado o treinamento na intensidade submáxima de alongamento ( $(\mathrm{S}, \mathrm{N}=14)$ e com o outro o treinamento na intensidade máxima de alongamento ( $T M, N=14$ ) sendo a escolha dos membros aleatória. Esse procedimento permitiu a redução de uma possível influência dos fatores relacionados à lateralidade ou dominância no efeito do treinamento.

Azevedo et al. ${ }^{(34)}$ não identificaram diferenças significativas da ADM após seis semanas para o grupo controle $(\mathrm{N}=12)$ utilizando o mesmo procedimento e mostrando uma baixa variabilidade na mensuração da ADM. Sendo assim um grupo controle não foi incluído no experimento. O mesmo indivíduo foi também submetido aos dois processos de treinamento, qualquer interferência iria refletir igualmente para ambos os membros inferiores testados.

\section{Análise dos dados}

Foi utilizada a análise de variança (ANOVA) two way 2x2 (intensidade $x$ treinamento) para identificar possíveis diferenças significativas da ADM no pré e pós-treinamento para as diferentes intensidades de alongamento. Para identificar onde se localizavam as diferenças recorreu-se ao teste post hoc Scheffé. Os dados foram analisados utilizando o programa Statistica 5.0. O nível de significância foi de $p<0,05$.

\section{RESULTADOS}

Os resultados da ANOVA two way demonstram uma diferença significativa apenas entre os valores médios da ADM do pré e pós-teste para o grupo de treinamento na intensidade máxima de alongamento. Os valores mínimos, máximos, médias e desvios-padrão da ADM na condição de pré e pós-teste para ambos os grupos estão apresentados na tabela 1. 
Tabela 1. Resultados do treinamento da flexibilidade na intensidade submáxima e máxima

\begin{tabular}{|c|c|c|c|c|c|c|c|c|}
\hline & \multicolumn{4}{|c|}{ ADM máxima pré-teste } & \multicolumn{4}{|c|}{ ADM máxima pós-teste } \\
\hline & Mínima & Máxima & Média & $d p$ & Mínima & Máxima & Média & $d p$ \\
\hline Treinamento submáximo (TS) & 41,0 & 79,0 & 68,9 & 14,0 & 41,5 & 86,5 & 71,0 & 14,4 \\
\hline Treinamento máximo (TM) & 46,0 & 84,5 & 68,0 & 10,5 & 55,5 & 88,5 & $74,0^{*}$ & 10,0 \\
\hline
\end{tabular}

* $p<0,05$ em relação ao pré-teste

A ANOVA two way indicou uma interação significativa entre os grupos (treinamento na intensidade máxima e submáxima de alongamento) e o teste (condição pré e pós) $(F=1.26 ; d f=8,62 ; p<0,007)$ (FIG. 3).

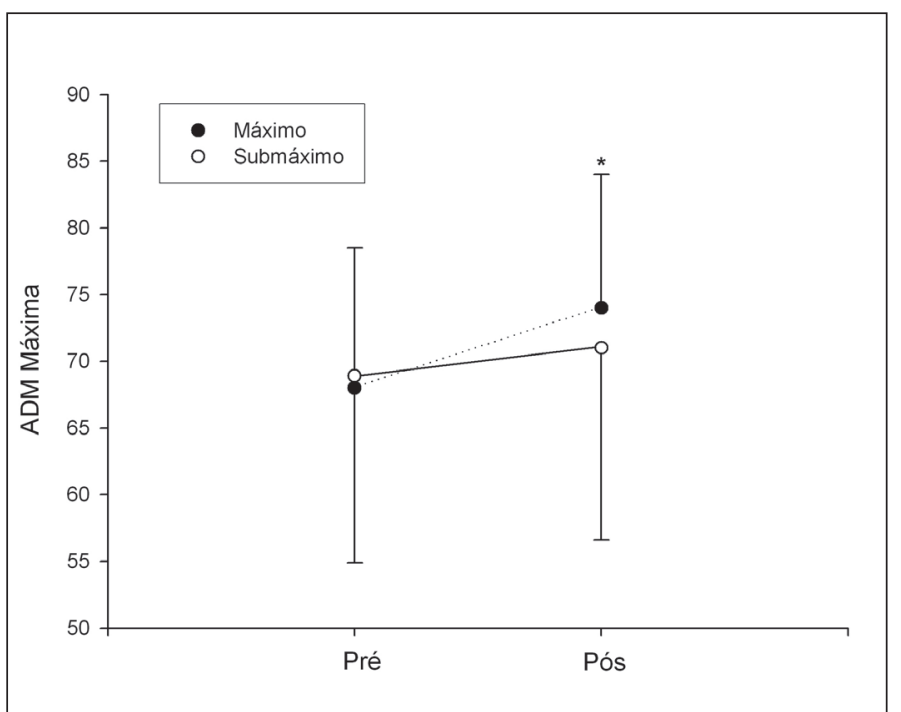

Figura 3. Amplitude de movimento máxima no pré e pós-treinamento para o grupo treinamento máximo (TM) e treinamento submáximo (TS).

* $p<0,05$ em relação ao pré-teste.

\section{DISCUSSÃO}

Os resultados corroboram aqueles da pesquisa de Marschall(24) e Sullivan et al. ${ }^{(16)}$, demonstrando um comportamento diferenciado da ADM para as intensidades de estímulo distintas, sendo que uma intensidade maior do alongamento foi mais efetiva para o aumento da ADM. O TS não demonstrou aumento significativo nos valores de ADM no pós-treinamento, diferentemente dos resultados do estudo de Marschall(24), embora a definição funcional para intensidade do TS seja a mesma. De acordo com os resultados relatados, quando intensidades máximas de alongamento são utilizadas, um efeito agudo significativo no aumento da ADM é possível. Em contrapartida, para intensidades submáximas de alongamento, o efeito agudo esperado é controverso e precisa ser mais investigado. Uma vez que técnicas de alongamento distintas podem ser influenciadas por diferentes mecanismos biomecânicos ${ }^{(30)}$ e neurofisiológicos ${ }^{(10)}$, os resultados obtidos com o uso da técnica passiva-estática no presente estudo e da técnica passiva-dinâmica na pesquisa de Marschall(24) demonstram a complexa relação entre a configuração da carga do treinamento e esses mecanismos. Além disso, é importante considerar que a carga de treinamento utilizada por Marschall(24) consistiu de 15 repetições do estímulo de alongamento, enquanto neste estudo foram apenas quatro repetições.
A configuração da carga de treinamento adotada neste estudo (quatro repetições de 15 segundos), baseou-se em pesquisas que demonstraram aumento da ADM com essa duração(30-33) e para esse número de repetições ${ }^{(30,32)}$, sendo esperado melhora da flexibilidade para os dois grupos de treinamento. Além disso, essa configuração aproxima-se bastante da caracterização dos treinamentos de flexibilidade recomendados na literatura e utilizados na prática esportiva. Provavelmente a intensidade adotada durante o alongamento no TS não foi capaz de resultar em uma tensão suficiente na musculatura a ponto de desencadear alterações que levassem a uma deformação significativa na unidade músculo-tendínea (UMT) detectável através do parâmetro ADM.

O comportamento da UMT em resposta ao alongamento passivoestático pode ser explicado principalmente pelas adaptações decorrentes das propriedades viscoelásticas ${ }^{(30,35)}$, neste caso, o relaxamento sob tensão. Esse mecanismo viscoelástico determina que quando uma UMT é alongada e mantida a um comprimento fixo, ocorre uma diminuição da tensão ao longo do tempo ${ }^{(30)}$. Essa redução da resistência passiva permite que a UMT seja mais facilmente deformada na segunda repetição, alcançando então um maior comprimento ${ }^{(30)}$. No entanto o protocolo experimental deste estudo não teve como objetivo investigar os possíveis mecanismos envolvidos na alteração aguda imposta pelo estímulo de alongamento e essa fundamentação permanece como uma explicação teórica.

Outra argumentação apresentada para alteração aguda da ADM é a tolerância ao alongamento. Magnusson et al. ${ }^{(36)}$ investigaram o efeito agudo do alongamento estático e dinâmico no aumento da ADM dos músculos posteriores da coxa. Os resultados não demonstraram alterações no comportamento viscoelástico da UMT que justificassem o aumento da ADM. Os resultados foram então associados ao aumento na tolerância ao alongamento. Entretanto, os mecanismos relacionados com as alterações na tolerância do alongamento permanecem desconhecidos. Uma possível abordagem para a discussão sobre a tolerância ao alongamento seria a Teoria do Portão de Controle da Dor. Baseandose nessa teoria, a estimulação de terminações nervosas nociceptivas presentes nas articulações e músculos tende a inibir a transmissão de sinais nociceptivos alterando a percepção da dor ${ }^{(37)}$. Possivelmente o estímulo de alongamento no TM ativa o portão de controle da dor de forma a aumentar a tolerância ao alongamento durante o treinamento alcançando uma ADM final maior quando comparada ao TS.

Apesar das medidas das intensidades de alongamento apresentarem-se confiáveis e precisas, não podemos descartar o caráter subjetivo do critério de determinação das intensidades. Isto significa que a relação entre a intensidade submáxima e máxima para cada voluntário não foi à mesma, ou seja, um indivíduo poderia treinar com uma intensidade submáxima que representaria 80\% da sua intensidade máxima, enquanto outro voluntário treinaria com um percentual diferente. Mesmo assim, todos treinaram em intensidades submáximas de acordo 
com a definição adotada no presente estudo. Estudos futuros deveriam considerar a relativização das intensidades entre os voluntários.

O resultado da ANOVA do presente estudo demonstrou interação significativa entre os grupos e o teste, indicando que a interpretação dos resultados referente ao aumento da ADM no treinamento de cada intensidade de alongamento não deve ser feita de maneira isolada.

Um importante resultado deste estudo é que intensidades de alongamento distintas podem representar cargas de treinamento específicas que irão provocar adaptações também específicas. Assim, a ampla variação da duração ótima do estímulo de alongamento para promover melhora na flexibilidade, de 10 a 120 segundos, descrita em outros estudos(6,21,33,35), poderia ser explicada por uma variação também do componente intensidade do estímulo no treinamento executado pelos voluntários desses estudos. Este mesmo raciocínio pode ser aplicado na discussão sobre a efetividade das diferentes técnicas de alongamento ${ }^{(16)}$.

\section{REFERÊNCIAS BIBLIOGRÁFICAS}

1. Chan SP, Hong Y, Robinson PD. Flexibility and passive resistance of the hamstrings of young adults using two different static stretching protocols. Scand J Med Sci Sports 2001; 11: 81-6.

2. Hardy L, Jones D. Dynamic Flexibility and Proprioceptive Neuromuscular Facilitation. Res Q Exerc Sport. 1986; 57: 150-3.

3. Hutton RS. Neuromuscular basis of stretching exercises. In Komi PV, editors. Strength and power in sport. London: Blackwell Scientific Publications, 1992; 29-38.

4. Roberts JM, Wilson K. Effect of stretching duration on a active and passive range of motion in the lower extremity. Br J Sports Med 1999; 33: 259-63.

5. Chagas MH, Shmidtbleicher D. Improvement of the range of motion and muscle passive tension during and after long-term stretching training. Proc 6th Annual Congress of European College of Sport Science 2001: 788.

6. Bandy WD, Iron JM. The effect of time on static stretch on the flexibility of the hamstring muscles. Phys Ther 1994; 74: 845-50.

7. Sady SP, Wortman M, Blanke D. Flexibility Training: Ballistic, Static or Proprioceptive Neuromuscular Facilitation. Arch Phys Med Rehabil 1982; 63: 261-3.

8. Holt LE, Travis TM, Okita T. Comparative study of three stretching techniques. Percep Mot Skills 1970; 31: 611-6.

9. Tanigawa MC. Comparison of the hold-relax procedure and passive mobility on increasing muscle length. Phys Ther 1972; 52: 725-35.

10. Moore MA, Hutton RS. Electromyographic investigation of muscle stretching techniques. Med Sci Sports Exerc 1980; 12: 322-9.

11. Wallin D, Ekblom B, Grahn R, Nordenborg T. Improvement of muscle flexibility: a comparison between two techniques. Am J Sports Med 1985; 13: 263-8.

12. Etnyre BR, Lee EJ. Chronic and acute flexibility of men and women using three different stretching techniques. Res Q Exerc Sport 1988; 59: 222-8.

13. Hartley-O'brien SJ. Six mobilization exercises for active range of hip flexion. Res Q Exerc Sport 1980; 51: 625-35.

14. Lucas RC, Koslow R, Comparative study of static, dynamic and proprioceptive neuromuscular facilitation stretching techiniques on flexibility. Percept Mot Skills 1984; 58: 615-8.

15. Wydra G, Bos K, Karisch G. Zur effektivität verschiedener Dehntechniken. Dtsch Z Sportmed 1991; 42: $386-534$.

16. Sullivan MK, Dejulia JJ, Worrell TW. Effect of pelvic position and stretching method on hamstring muscle flexibility. Med Sci Sports Exerc 1992; 24: 1383-9.

17. Schönthaler S, Ott H, Schwaz L, Kindermann W. Effects of different stretching methods on the maximum range of motion (ROM). J Sports Med 1996; 17: 74.

18. Chagas MH, Shmidtbleicher D. Effect of increase of the range on muscle strength performance. Proc 6th Annual Congress of European College of Sport Science, Cologne 2001; 1040.

19. Willy RW, Kyle BA, Moore SA, Chleboun GS. Effect of cessation and resumption of static hamstring muscle stretching on joint range of motion. J Orthop Sports Phys Ther 2001; 31: 138-44.
Através do presente estudo conclui-se que o comportamento da ADM entre o pré-teste e pós-teste é diferente para as intensidades máxima e submáxima de alongamento, após uma sessão de treinamento utilizando quatro repetições com duração de 15 segundos. Sendo indicado para o treinamento da flexibilidade a utilização da intensidade máxima de alongamento quando o objetivo é o aumento significativo da ADM. O efeito não significativo da intensidade submáxima na ADM verificado no presente estudo não pode ser generalizado. É necessário investigar se a utilização da intensidade submáxima de alongamento dentro de outras configurações da carga de treinamento, por exemplo, maiores durações e número de repetições do estímulo, poderia promover aumento na ADM.

Todos os autores declararam não haver qualquer potencial conflito de interesses referente a este artigo.
20. Bandy WD, Iron JM, Briggler M. The effect of time and frequency of static stretching on flexibility of the hamstring muscles. Phys Ther 1997; 77: 1090-6.

21. Borms J, Van Roy P, Santens JP, Haentjens A. Optimal duration of static stretching exercises for improvement of coxofemoral flexibility. J Sports Sci 1987; 5: 39-47.

22. Youdas, JW, Krause DA, Egan KS, Therneau TM, Laskowski ER. The effect of static stretching of the calf muscle-tendon unit on active ankle dorsiflexion range of motion. J Orthop Sports Phys Ther 2003; 33: 408-16.

23. Bandy WD, Iron JM, Briggler M. The effect of static stretch and dynamic range of motion training on the flexibility of the hamstring muscles. J Orthop Sports Phys Ther 1998; 27: 295-300.

24. Marschall F. Wie beeinflussen unterschiedliche Dehnintensitäten kurzfristig die Veränderung der Bewegungsreichweite. Dtsch Z Sportmed 1999; 50: 5-9.

25. Tan B. Manipulating resistance training program variables to optimize maximum strength in men: a review. J Strength Cond Res 1999; 13: 289-304.

26. American College of Sports Medicine (ACSM) Position stand on progression models in resistance training for healthy adults. Med Sci Sports Exerc 2002; 34: 364-80.

27. Kraemer WJ, Ratamess NA. Fundamentals of resistance training: progression and exercise prescription Med Sci Sports Exerc 2004; 36: 674-88.

28. Netto CM, Júnior LAM, Chagas MH, Confiabilidade do teste de extensão de joelho modificado. Anais X Congresso Brasileiro de Biomecânica, Belo Horizonte, 2003; 2: 149-52.

29. Bergamini JC, Bhering EL, Gomes EC, Menzel HJ, Chagas MH. Confiabilidade de duas diferentes intensidades de alongamento no teste de extensão do joelho modificado. XI Congresso Brasileiro de Biomecânica 2005: .

30. Taylor DC, Dalton JD, Seaber AV, Garrett W.E. Viscoelastic properties of muscle tendon units: the biomechanical effects of stretching. Am J Sports Med 1990; 18: 300-9.

31. Zakas, A. The effect of stretching duration on the lower-extremity flexibility of adolescent soccer players. J Bod Mov Ther, 2005; 9: 220-5.

32. Zakas, A.; Balaska, P.; Grammatikopoulou, M.G.; Zakas, N.; Vergou, A. Acute effects of stretching duration on the range of motion of elderly women. J Bod Mov Ther, 2005; 9: 270-6.

33. Madding SW, Wong JG, Hallum A, Medeiros JM. Effect of duration of passive stretch on hip abduction range of motion. J Orthop Sports Phys Ther 1987; 8: 409-16.

34. Azevedo DC, Damasceno ACD, Gianeli PC, Chagas MH. Influência do posicionamento do tornozelo sobre a melhora da flexibilidade dos músculos isquiotibiais. Anais do X Congresso Brasileiro de Biomecânica, Belo Horizonte, 2003; 2: 434-7.

35. Gajdosik RL. Effects of static stretching on the maximal length and resistance to passive stretch of short hamstring muscles. J Orthop Sports Phys Ther 1991; 14: 250-5.

36. Magnusson SP, Aagaard P, Simonsen EB, Bojsen-Moller, F. A Biomechanical Evaluation of Cyclic and Static Stretch in Human Skeletal Muscle. Int J Sports Med 1998; 19: 310-6.

37. Jessell TM, Kjelly DD. Percepção e dor. In: Kandel ER, Schwartz JH, Jessell TM, editores. Princípios de Neurociência. Barueri, SP: Manole, 2003; 472-91. 